\title{
Erratum to: Cetyltrimethylammonium Bromide Capped 9-Anthraldehyde Nanoparticles for Selective Recognition of Phosphate Anion in Aqueous Solution Based on Fluorescence Quenching and Application for Analysis of Chloroquine
}

Prasad G. Mahajan • Netaji K. Desai •

Dattatray K. Dalavi • Dhanaji. P. Bhopate •

Govind B. Kolekar • Shivajirao R. Patil

Published online: 31 October 2014

(C) Springer Science+Business Media New York 2014

Erratum to: J Fluoresc

DOI 10.1007/s10895-014-1451-7

The original version of this article unfortunately contained a mistake. Equation (4) was incorrect. The correct equation is shown below:

$\mathrm{R}_{\mathrm{d}}=\mathrm{K}_{\mathrm{d}} \cdot \theta$ 10.1007/s10895-014-1451-7.

P. G. Mahajan • N. K. Desai • D. K. Dalavi • D. P. Bhopate •

G. B. Kolekar S S. R. Patil $(\bowtie)$

Fluorescence Spectroscopy Research Laboratory, Department of

Chemistry, Shivaji University, Kolhapur 416 004, Maharashtra, India

e-mail: srp_fs1@rediffmail.com

S. R. Patil

e-mail: srp_chem@unishivaji.ac.in 